\title{
Critical Design Issues for Space Borne Polarimetric Radiometry
}

\author{
Peter Gaiser, Gene Poe and Karen St. Germain \\ Naval Research Laboratory \\ Code 7223, Washington DC 20375 \\ (202)767-8253;FAX (202)767-9194 \\ gaiser@vaximg.nrl.navy.mil;poe@vaximg.nrl.navy.mil; ksaint@ultrimg.nrl.navy.mil
}

\begin{abstract}
Polarimetric microwave radiometry has received much attention in recent years as a potential tool for measuring the ocean surface wind vector. Several airborne sensors have been built and collected valuable data. This paper describes some of the important design issues that must be addressed to deploy successfully a satellite-based polarimetric microwave radiometer.
\end{abstract}

\section{INTRODUCTION}

Space borne microwave radiometry has a strong heritage based on the Special Sensor Microwave/Imager (SSM/I) operated by the Defense Meteorological Space Program (DMSP). Over the past ten years, five SSM/I's have successfully provided reliable passive microwave data for retrieving environmental parameters such as wind speed, sea ice concentration and age, and integrated atmospheric water vapor. One parameter that has not been provided by microwave radiometers is the wind direction. However, recent research results demonstrated that [1], [2] usable direction information can be obtained by measuring the crosscorrelation of vertically and horizontally polarized brightness temperatures, $T_{\nu}$ and $T_{h}$ respectively. This yields the third and fourth parameters of the Stokes vector, $I_{s}$, defined by [3] as,

$$
I_{\mathrm{s}}=\left[\begin{array}{c}
I \\
Q \\
U \\
V
\end{array}\right]=\left[\begin{array}{c}
T_{\mathrm{v}} \\
T_{\mathrm{h}} \\
T_{45}-T_{-45} \\
T_{\mathrm{lc}}-T_{\mathrm{rc}}
\end{array}\right]=\left[\begin{array}{c}
\left\langle E_{\mathrm{v}} E_{\mathrm{v}}^{*}\right\rangle \\
\left\langle E_{\mathrm{h}} E_{\mathrm{h}}^{*}\right\rangle \\
2 \operatorname{Re}\left\langle E_{\mathrm{v}} E_{\mathrm{h}}^{*}\right\rangle \\
2 \operatorname{Im}\left\langle E_{\mathrm{v}} E_{\mathrm{h}}^{*}\right\rangle
\end{array}\right]
$$

$U$ and $V$ are odd functions of the relative wind direction, while, $T_{v}$ and $T_{h}$ are even functions.

Several recent aircraft experiments have investigated the ocean surface microwave scattering and emission, and how these apply to wind vector retrievals [1], [2]. The brightness temperature variations with azimuth angle are clearly evident, where peak-to-peak brightness temperature modulation appears to vary from about $5 \mathrm{~K}$ in higher winds $(>15 \mathrm{~m} / \mathrm{s})$ to less than $1 \mathrm{~K}$ at low wind speeds $(<3 \mathrm{~m} / \mathrm{s})$.

The success of the aircraft campaigns has given impetus to developing a space borne polarimetric microwave radiometer. The Naval Research Laboratory (NRL), with support from Operational Navy, the National Polar-orbiting Operational Satellite System (NPOESS) and the Air Force Space Test Program is developing the WindSat satellite-based instrument. WindSat builds on the heritage of SSM/I, but incorporates polarimetric channels. In addition, because of the small wind direction signal, WindSat performance requirements are generally stricter than for SSM/I and other satellite-based radiometers flown or being developed. Some areas of design that require special attention are receiver linearity, antenna cross-polarization and spacecraft attitude control.

\section{RECEIVER LINEARITY}

The WindSat design is based on measuring the total power in the six primary polarization, vertical, horizontal, $\pm 45^{\circ}$ linear, left circular and right circular polarization. The third and fourth Stokes parameters, $T_{u}$ and $T_{4}$, are derived by taking the differences between the appropriate polarizations. This technique simplifies the design and calibration complexity. However, special attention must be paid to ensure that calibration errors in each channel be common mode in order to cancel in the differencing process. Some errors will be unique to a given receiver channel, and thus, will not subtract. These errors must be minimized. The WindSat system uses a warm load and a cold sky reflector as calibration sources, which should present largely common mode errors if well designed and fabricated. The largest contributor to the independent calibration errors is the receiver non-linearity, which is dominated by the detector diode. To minimize the residual non-linearity error $(<0.1 \mathrm{~K})$ in $T_{u}$ and $T_{4}$, the diodes in a given receiver pair must be matched in non-linearity to within approximately $0.06 \%$. Alternatively, one can use diodes that are extremely linear over the operating range. Laboratory testing during the WindSat design phase confirmed that matching the diode output impedance greatly improves the linearity [4], resulting the total non-linearity bias being less than $0.1 \mathrm{~K}$. 


\section{ANTENNA CROSS-POLARIZATION}

Another technical consideration that must not be overlooked is antenna performance. A polarimetric microwave radiometer measures the Stokes parameters. Upon reviewing the Stokes vector equation, one readily sees that these parameters quantify the polarization purity and coupling of a scene. Therefore, it is important to minimize the polarization coupling in the antenna and orthomode transducer (OMT). It is also necessary to understand the effective antenna patterns of the Stokes parameters. Typical Space Borne imaging radiometers such as WindSat and SSM/I use offset parabolic reflector antennas for the high beam efficiency. However, this type of antenna has inherent cross-polarization that inversely depends on the $f / D$ ratio. The cross-polarization and antenna beam efficiencies must be calibrated and incorporated into an antenna pattern correction (APC) as part of the data processing. On dual-polarization systems, the APC has been a two-by-two matrix. For a polarimetric radiometer, this becomes a four-by-four matrix. This APC process is express by the following equation

$$
\left[\begin{array}{c}
T_{v}^{\prime} \\
T_{H}^{\prime} \\
T_{U}^{\prime} \\
T_{4}^{\prime}
\end{array}\right]=\left[\begin{array}{llll}
a_{v v} & a_{h v} & a_{u v} & a_{4 v} \\
a_{v h} & a_{h h} & a_{u h} & a_{4 h} \\
a_{v u} & a_{h u} & a_{u u} & a_{4 u} \\
a_{v 4} & a_{h 4} & a_{u 4} & a_{44}
\end{array}\right]\left[\begin{array}{c}
T_{V} \\
T_{H} \\
T_{U} \\
T_{4}
\end{array}\right]
$$

where $T$ is the true scene brightness temperature and $T^{\prime}$ is the measured brightness at the output of the antenna system. $\underline{a}_{\underline{y}}$ is the vertical co-polarized signal; $a_{p q}$ represents the coupling of energy from polarization $p$ into polarization $q$. The lower left corner of this matrix is very important because it represents coupling of $T_{v}$ and $T_{h}$ into $T_{u}$ and. The former two are on the order of 100-200 K, whereas the latter two are approximately $\pm 2 \mathrm{~K}$. Therefore, $20 \mathrm{~dB}$ of cross polarization could easily cause an error of $100 \%$. Cross-polarization coupling for a polarimetric radiometer must be calibrated to better than $30 \mathrm{~dB}$.

To better understand the antenna behavior for a polarimetric radiometer, NRL has developed a model to produce full antenna patterns for the Stokes vector for offset parabolic reflectors. This antenna model produces the following results:

- Co- and cross-polarization antenna patterns and beam efficiencies for vertical polarization, horizontal polarization, and the third and fourth Stokes parameters (U and $\mathrm{V}$ ).

- The antenna pattern and beam efficiency calculations account for feed location relative to the focal point and aperture illumination

- Polarization rotation for feeds not located at the focal point.
This model provides essential information for the design of an antenna that will adequately measure the Stokes parameters. It can also quantify the performance of existing antennas, such as the SSMIS, to evaluate their potential for passive polarimetry. For example, the WindSat design uses a multiple feedhorns in an array around the reflector focal point. Figure 1 shows the H-plane co- and cross-polarization antenna patterns for vertical polarization and the fourth Stokes parameter, $T_{4}$, at $10.7 \mathrm{GHz}$ located at $(x=0.122$ in, $y=-4.34$ in) relative to the focal point. Relative to the satellite motion, the $x$ axis is along track and the $y$-axis is across track. The fact that the feed is not in the focal point accounts for the asymmetry of the beams, and causes increased coupling of $\mathrm{V}$ and $\mathrm{H}$ polarizations into the third and fourth Stokes parameters.
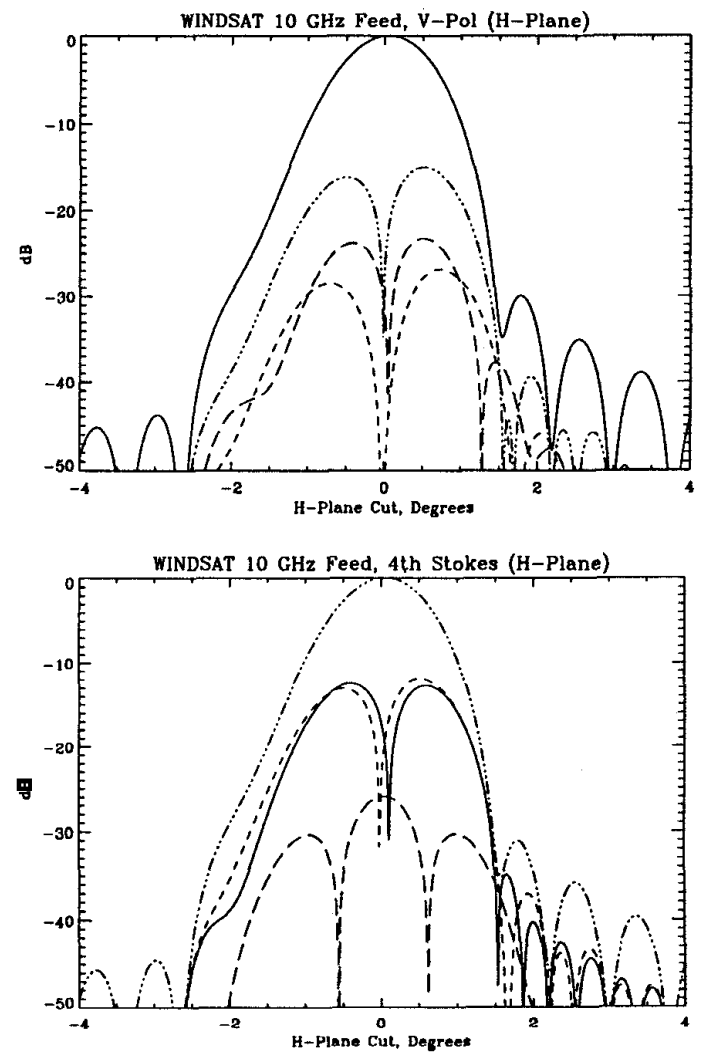

Figure 1. Antenna patterns for vertical polarization and the fourth Stokes parameter, $T_{4}$, at $10.7 \mathrm{GHz}$ for the WindSat antenna. 


\section{SPACECRAFT ATTITUDE}

Cross-polarization errors can be introduced by the spacecraft attitude in addition to the antenna properties. The integration of the radiometer antenna system onto the spacecraft defines the polarization basis of the radiometer payload. In the ideal world, this would be perfectly aligned with the polarization basis of the Earth. However, spacecraft pitch, roll and yaw cause these two bases to be misaligned, introducing a polarization rotation error. Additional error sources include misalignment of the antenna with respect to the spacecraft and gravity off-loading on orbit. Care in design, integration and modeling can minimize the impact of this latter group. The spacecraft attitude can be addressed in two ways: control and determination. The WindSat system requires maintaining the misalignment between the radiometer and the Earth to approximately $0.05^{\circ}$ or less. Controlling the spacecraft to do this is costly. The alternative is to measure the spacecraft attitude and correct for misalignments. Star trackers and inertial reference units are capable of determining the spacecraft attitude to much better than $0.05^{\circ}$. These measurements are then converted into the polarization rotation error and a correction is applied. Polarizations may be transformed with the following matrix equation, where $\varphi$ is the polarization rotation angle [3].

$$
\left[\begin{array}{l}
T_{A} \\
T_{B} \\
T_{C}
\end{array}\right]=\left[\begin{array}{ccc}
\cos ^{2} \varphi & \sin ^{2} \varphi & \frac{1}{2} \sin \varphi \\
\sin ^{2} \varphi & \cos ^{2} \varphi & -\frac{1}{2} \sin \varphi \\
-\sin 2 \varphi & \sin 2 \varphi & \cos 2 \varphi
\end{array}\right]\left[\begin{array}{l}
T_{V} \\
T_{H} \\
T_{U}
\end{array}\right]
$$

This is a very robust correction that will allow adequate correction of the errors to the level of knowledge of the attitude determination system. Circular polarization is insensitive to polarization rotation.

\section{CONCLUSION}

The ocean surface wind vector plays an important role in meteorology, oceanography, climatology and military operations. Airborne polarimetric microwave radiometers have demonstrated the capability to retrieve the ocean wind vector with the radiometric Stokes vector. Space Borne systems have excellent potential to measure the ocean wind vector from space. However, to do so successfully, the radiometer designers must build on the heritage of SSM/I and improve on the SSM/I performance considerably. Small errors that were negligible to previous systems present considerable problems to polarimetric radiometers attempting to measure the wind direction because of the small directional signal. Design areas that require particular attention include antenna cross-polarization; diode linearity and matching; and spacecraft attitude determination. While very challenging, the improved performance required of a polarimetric microwave radiometer is achievable.

[1] Yueh, S. H., W.J. Wilson, F. K. Li, S.Y. Nghiem and W. B. Ricketts, "Polarimetric measurements of the sea surface brightness temperature using an aircraft $\mathrm{K}$ band radiometer", IEEE Trans. Geoscience and Remote Sensing, vol. 33, no. 1, pp 85-92, 1995.

[2] P. Chang, P. Gaiser, K. St. Germain, and L.Li, "MultiFrequency polarimetric microwave ocean wind direction retrievals," Proceedings of the International Geoscience and Remote Sensing Symposium 1997, Singapore, 1997.

[3] L. Tsang, J. A. Kong, and R. T. Shin, Theory of Microwave Remote Sensing, John Wiley and Sons, 1985.

[4] C. Hoer, K. Roe and C. Allred, "Measuring and minimizing diode detector nonlinearity," IEEE Trans.on Instrumentation and Measurement, vol. IM-25, no. 4, December, 1976. 